\title{
TIME REQUIRED FOR LEVEL SPEED MEASUREMENT
}

Mr. Lush is to be thanked for his paper "A Note on the Time Required to Make a Level Speed Measurement with a Turbine-Jet Aircraft" (October 1950 JOURNAL). He should be congratulated for bringing to notice an interesting aspect of modern aeroplane behaviour. He attacks the problem in a most elegant mathematical fashion, but I must admit that when I read his paper through I was left with a vague unsatisfied feeling-it was only after several patient re-readings that I traced my disquiet to his factor $Y$. He states that variations in this are responsible for about half the total magnitude of the effect under consideration on jet aircraft. Apparently, the values of $Y$ have to be read off from curves which were not presented.

As one whose predominant interest is in engines, $I$ feel that more information on this side would help to complete the picture. Accordingly, I have tried to deduce succinctly the variations in $Y$ from fundamental relations for gas turbines.

The following symbols and subscripts are employed. To avoid confusion with temperatures, Mr. Lush's $T$ for thrust has been converted to $X$ in the present notation. $T$ stands for temperature, $P$ for pressure (lb./in. ${ }^{2}$ ), $A$ for air mass flow (lb./sec.), $V$ for aircraft speed in $\mathrm{ft}$./sec., $N$ for engine r.p.m., $v$ for gas speeds and $f$ for area $\left(\mathrm{ft}^{2}{ }^{2}\right)$. Subscripts are used to describe different stations in the enginesee Fig. 1.

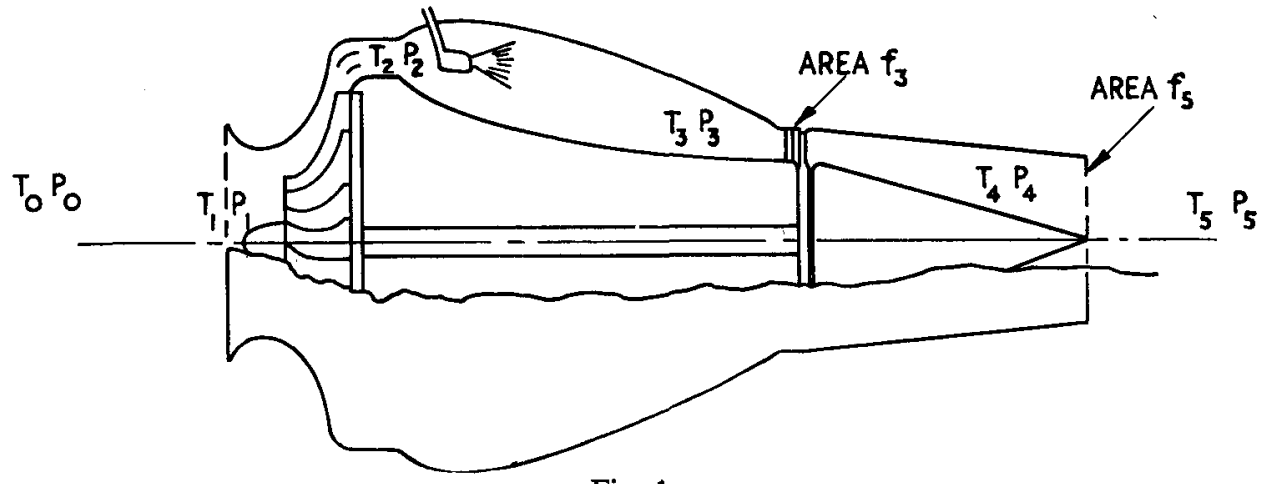

Fig. 1.

Mr. Lush's expression was

$$
Y=(V / X) d X / d V \text {. }
$$

From momentum considerations, the thrust of a jet engine can be expressed as

$$
X=(A / g)\left(1.015 v_{5}-V\right)+f_{5}\left(P_{5}-P_{0}\right) .
$$

(The constant 1.015 makes allowance for the average value of the additional mass of the fuel which is burnt.)

The last term comes into play only when the flow through the propulsive nozzle becomes supersonic; under subsonic conditions $\boldsymbol{P}_{5}=\boldsymbol{P}_{0}$ and the term vanishes.

For the variation of the air mass flow through the engine, the examination will be restricted to conditions of engine speed, such that sonic relations may be assumed to exist through the turbine nozzle guide vanes without introducing appreciable error. This applies on very nearly all present engines down to half-thrust conditions at least, and usually considerably below. (N.B.-Ram pressure ratios also influence matters.)

Hence

$$
A=\lambda f_{3} P_{2} / \sqrt{ } T_{3} . \quad \text {. . . . . . }
$$

(The value of $\lambda$ is approximately 0.37 in self-consistent units.) 
Moreover, the blower pressure ratio may be expressed as

$$
\frac{P_{2}}{P_{1}}=\left\{1+\frac{\theta N^{2}}{T_{1}}\right\}^{\frac{\gamma}{\gamma-1}} ; \frac{P_{1}}{P_{0}}=\left\{1+\frac{\xi V^{2}}{T_{0}}\right\}^{\frac{\gamma}{\gamma-1}}
$$

where $\theta$ and $\xi$ are constants.

Therefore

$$
\frac{P_{2}}{P_{0}}=\left\{1+\frac{\xi V^{2}}{T_{0}}+\frac{\theta N^{2}}{T_{0}}\right\}^{\frac{\gamma}{\gamma-1}} . . .
$$

Sonic flow in propulsive nozzle

Differentiate now between the case of sonic flow in the propulsive nozzle and subsonic conditions. Considering the former case, it follows from general laws. that the values of $T_{3}$ and $v_{3}$ are independent of the aircraft velocity $V$, and the ratio $P_{5} / P_{0}=k P_{1} / P_{0}$. The constant $k$ will have a fractional value unless sonic flow is encountered in the propulsion nozzle under high r.p.m. conditions at zero forward speed. This is not generally the case.

From equations (1), (2), (3) and (4) an expression for $Y$ may be obtained by suitable arrangement and simple differentiation:-

$$
Y=\frac{\frac{1}{T_{0}}\left[\frac { \lambda } { g } \left\{\left(1+\frac{\theta N^{2}}{T_{0}}+\frac{\xi V^{2}}{T_{0}}\right)^{\frac{1}{\gamma-1}} \frac{2 \xi V^{2} \gamma}{\gamma-1}\left(1.015 v_{5}-V\right)-\right.\right.}{\left.\left.-T_{0} V\left(1+\frac{\theta N^{2}}{T_{0}}+\frac{\xi V^{2}}{T_{0}}\right)^{\frac{\gamma}{\gamma-1}}\right\}+\frac{2 \xi V^{2} \gamma}{\gamma-1} T_{3}{ }^{\frac{1}{f_{5}}} \frac{f_{3}}{f_{3}}\left(\frac{P_{1}}{P_{0}}\right)^{\frac{1}{\gamma}}\right]}
$$

(It should be noted that $P_{2} / P_{0}$ and $P_{1} / P_{0}$ are variables and the respective functions have been differentiated with respect to $V$.)

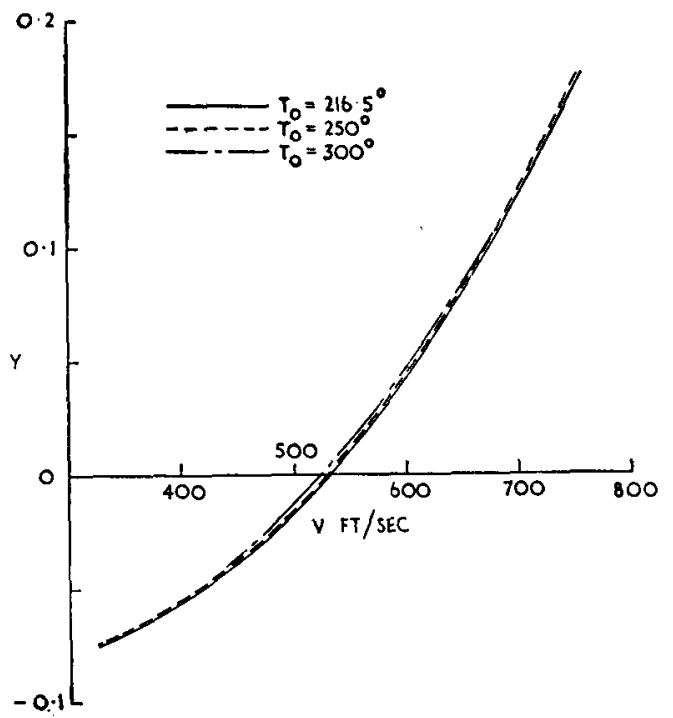

Fig. 2. 


\section{CORRESPONDENCE}

This expression is solely dependent, with respect to changes in height, on the temperature $T_{0}$ and not on pressures. It is too complex to appreciate as it stands and curves have been drawn (in Fig. 2) for three specific cases. It will be seen that all the curves lie very closely together although this is not obvious from the equation (5). It should be noted that in the calculations constant engine speed (maximum) operation has been assumed. A family of curves could be plotted for different engine speeds, and then it would be necessary to know the relationship between aircraft velocity in level flight and engine speed to select the correct parameter to use.

\section{Subsonic flow}

In this case the last term of equation (2) may be neglected. Substituting from (3) and differentiating:-

$$
Y=\frac{V}{P_{2}} \frac{\partial P_{2}}{\partial V}-\frac{V}{2 T_{3}} \frac{\partial T_{3}}{\partial V}+\frac{V}{1.015 v_{5}-V}\left(1.015 \frac{\partial v_{5}}{\partial V}-1\right) \quad . \quad .
$$

Unfortunately it is not possible to show within the confines of this letter how this expression will vary because all three of the terms in the equation are of the same order of magnitude and great accuracy is required in the individual terms if the resultant figure, which is a difference, is not to be very inaccurate.

O. N. LAWRENCE, Associate Fellow.

I would like to thank Mr. Lawrence for his congratulations and also to offer congratulations myself to the printers for printing his equation (5).

As my paper was concerned with the reactions of engine characteristics on the behaviour of the aircraft I thought it proper to omit from it any examination of the mechanism of these characteristics. I am interested in the latter subject, but it only concerns me because any theory which would lead to reliable generalisations about $Y$ and the variation of $Y$ and net thrust with engine speed would be of great value in performance testing jet aircraft. I am particularly concerned with the climb case, when high air mass flow is combined with a relatively low air speed. Can Mr. Lawrence help here?

It would seem that in deducing equation (5) it has been assumed that intake and compressor efficiencies are independent of air speed. Is this assumption generally applicable? Also, if allowance is to be made for fuel mass flow in the exhaust is it permissible to neglect cooling drag?

K. J. LUSH 